\title{
Socio-economic differences in adolescents' breakfast eating, fruit and vegetable consumption and physical activity in Ghana
}

\author{
David Doku ${ }^{1, *}$, Leena Koivusilta ${ }^{2}$, Susanna Raisamo ${ }^{1}$ and Arja Rimpelä ${ }^{1}$ \\ ${ }^{1}$ School of Health Sciences, University of Tampere, FI-33014 University of Tampere, Tampere, Finland: \\ ${ }^{2}$ Department of Social Research, Faculty of Social Sciences, University of Turku, Turku, Finland
}

Submitted 22 February 2011: Accepted 19 September 2011: First published online 27 October 2011

\begin{abstract}
Objective: Few studies have investigated the association between adolescents' socio-economic status (SES) and health behaviours in developing countries. Socio-economic differences in breakfast eating, fruit and vegetable consumption and physical activity were investigated among Ghanaian adolescents.

Design: A school-based survey of 12-18-year-olds ( $n$ 1195, response rate $89 \cdot 7 \%$ ) was conducted in 2008. Logistic regression analyses were applied to study the associations between breakfast eating, fruit and vegetable consumption and physical activity, and SES.

Setting: Southern Ghana, West Africa.

Subjects: School pupils aged 12-18 years.

Results: Of all adolescents, $31 \%$ took breakfast less than $4 \mathrm{~d} /$ week, over half (56\%) and $48 \%$, respectively, rarely ate fruits and vegetables. Younger adolescents (12-15-year-olds) consumed fruits and vegetables frequently compared with older ones (16-18-year-olds). Boys were more likely to participate in physical activity than girls. The probability of frequent breakfast eating was higher in adolescents from more affluent backgrounds than in those from less affluent ones. Compared with those whose mothers were illiterate, both tertiary and primary maternal educational attainment increased the probability of frequent fruit intake. Similarly, tertiary educational attainment increased the likelihood of frequent vegetable intake. Compared with those with unemployed mothers, mothers' low employment grade was related to higher probability of frequent fruit intake. High school performance was associated with frequent fruit intake, whereas high or medium school performance increased the likelihood of vegetable intake compared with low school performance.

Conclusions: Interventions are needed to improve breakfast intake, fruit and vegetable consumption and physical activity in order to prevent degenerative diseases among Ghanaian adolescents.
\end{abstract}

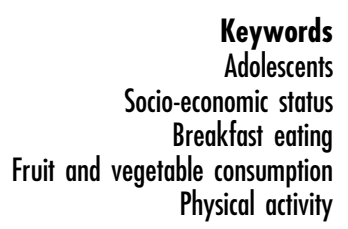

In Ghana, as in many African countries, there is rapid urbanisation and a gradual shift from an agrarian economy towards a service-based economy. This has resulted in a corresponding swift shift from intensive physical labour to non-manual labour, the adoption of some lifestyle behaviours such as use of cars for transportation, and the proliferation of Internet cafés, cinemas, fast-food restaurants and all kinds of sedentary behaviours that are mostly associated with urbanisation or Western culture. It is worrying that in addition to the numerous infectious diseases, including e.g. HIV/AIDS and malaria, healthdamaging behaviours and lifestyles - which hitherto were common only in Western societies - are now at high rates in many African countries ${ }^{(1,2)}$.
Breakfast eating, fruit and vegetable consumption and physical activity are important health behaviours, especially during the developmental stages of life ${ }^{(3-7)}$. Research has shown that regular intake of breakfast increases the possibility of a balanced diet and improves overall nutritional status and nutritional density, particularly during the early phases of life ${ }^{(5)}$. Studies have shown that fruit and vegetable intake may decrease cancer incidence by $20 \%$, protect against CVD, enhance bone mineral and can lead to lower urinary calcium output ${ }^{(8,9)}$. Lack of physical activity is a determinant of poor health and as well increases the risk of chronic lifestyle diseases such as diabetes, overweight, obesity, hypertension and osteoporosis. In addition, lack of physical activity may be 
associated with psychological disorders and antisocial behaviours at different life stages ${ }^{(3,4)}$. Furthermore, there is evidence that adolescents who participate in physical activity are likely to continue to do so into adulthood ${ }^{(10)}$.

Studies that will lead to a greater understanding of the factors that influence breakfast eating, fruit and vegetable intake and physical activity in adolescence are therefore crucial in developing programmes and policies that seek to prevent degenerative diseases and promote physical activity and healthy life in adulthood. Inequalities in risk behaviours such as breakfast eating, fruit and vegetable consumption and physical inactivity can lead to socioeconomic differences in health. The onset of these health behaviours is in childhood and their health consequences can extend through adolescence into adulthood ${ }^{(11)}$. The relationship between adolescents' socio-economic status (SES) and these behaviours has been extensively studied in Western countries. A review conducted by Hanson and Chen ${ }^{(12)}$ found that adolescents from low-SES backgrounds were at higher risk of poor diet and physical inactivity. Not all studies have found this relationship; however, as each study used a different SES measure, this could account for the inconsistency in the findings. There is hardly any study from African countries assessing SES differences in breakfast eating, fruit and vegetable intake or physical activity, thus creating bias in the literature about what is known on the subject in the region. To our knowledge, there was only one study from South Africa that investigated the socio-economic differences in physical activity among 9-year-olds ${ }^{(13)}$. McVeigh et al. $^{(13)}$ used housing characteristics, household assets, parental marital status, parental education and ethnic background as measures of SES and reported that children from higherSES backgrounds were more physically active than those from lower-SES backgrounds.

The present study seeks to investigate the prevalence and socio-economic differences in breakfast eating, fruit and vegetable consumption and physical activity among Ghanaian adolescents using multiple socio-economic indicators.

\section{Methods}

\section{Participants and procedure}

The present study is based on a cross-sectional survey conducted from June to August 2008 on health behaviours and lifestyles of school-aged adolescents in three regions in Ghana. Thirty schools were randomly sampled, ten per region, from Eastern, Greater Accra and Volta regions. The Ghana Education Service's School Health Programme register of schools in the country was the source used in sampling the schools. The schools were selected so that they comprised four public Junior High Schools, two private Junior High Schools, three public Senior High Schools and one private Senior High School in each region in order to reflect the school types in Ghana. In each school, about fifty students were selected at random. All students whose names were found in the class attendance registers of the selected classes were eligible to participate in the survey. The school enrolment rate in Ghana is $78.8 \%$ for Junior High Schools ${ }^{(14)}$, but information on enrolment rates for Senior High Schools was not available. Written informed consents were obtained from 18-year-old adolescents and parents of those under 18 years old who voluntarily agreed to participate in the study.

The eight-page questionnaire contained modules on substance use, sociodemographic factors, food habits, sleep, physical activity, sexual health, general health practices and future health behaviour. It was anonymous and self-administered and tested with an initial pilot sample of fifty children in three schools. It was designed to exclude any information that would reveal the identity of the participants. One trained supervisor from the research team was assigned to each classroom during the answering. The survey commenced simultaneously in all of the participating classes in a given school. Participants were asked to drop their questionnaires in an envelope placed in front of the class on completion. The response rate was $89 \cdot 7 \%$ (based on attendance records on the survey days). Out of the 1566 respondents who completed the questionnaire, 1195 (all 12-18-year-old students) were included in the present study. The study protocol was approved by the ethical committee of the Ghana Health Service Research Unit in Accra, Ghana.

\section{Indicators of socio-economic status}

\section{Familial indicators}

A material affluence scale of five categories ('poorest', 'poor', 'average', 'affluent' and 'most affluent') was used based on previous work ${ }^{(15)}$ done by the research group of the present paper. The items covered aspects of material circumstances: household assets (fridge/freezer, television, radio, computer and car) and housing characteristics (type of house and building material, and availability of electricity). Material affluence mirrors the lack of or availability of the resources and goods necessary for decent living in relation to what is generally available in the society ${ }^{(10)}$. Various kinds of scales measuring material affluence have been constructed to capture the amount of these kinds of resources available in families ${ }^{(15,17)}$. The items of the scales cover the key aspects of wealth as well as the material circumstances of the family.

Family structure was measured in four categories: 'nuclear family', 'both parents alive but not living together', 'only one parent alive' or 'both parents dead'.

\section{Parental indicators (parental education and occupation)}

Father's, mother's or guardian's highest level of education was categorised into 'illiterate', 'primary education', 
'secondary education' and 'tertiary education' according to the classification of the Ghanaian educational system. Parental occupational status was measured by each respondent reporting his/her father's, mother's or guardian's occupation or employment status. The responses were then condensed into six categories: (i) unemployment; (ii) grade $\mathrm{A}$, chief in rank; (iii) grade $\mathrm{B}$, professional and managerial; (iv) grade $\mathrm{C}$, professional non-managerial; (v) grade D, skilled manual; and (vi) grade E, unskilled manual, according to the occupational classification in the Ghanaian civil service (Head of Civil Service, 2000, personal communication). None of the respondents fell into the A category. We combined grades $\mathrm{B}$ and $\mathrm{C}$ as 'high grade' and grades D and E as 'low grade'.

\section{Level of urbanisation}

Level of urbanisation was based on the location of the school: schools located in the regional and district capitals were categorised as 'urban' and all others as 'rural'.

\section{Adolescent's own social position}

We used indicators that measured the young people's present social position and at the same time anticipated their future socio-economic position.

Adolescents were asked to indicate their school performance in the previous term's examination by ticking one of the following responses: 'excellent', 'very good', 'good', 'average' and 'poor'. These were coded into three categories: 'excellent' and 'very good' as 'high'; 'good' as 'middle'; and 'average' and 'poor' as 'low'.

Adolescents were asked to indicate their plans after graduation from the current level of schooling, choosing from the following responses: 'continue schooling', 'learn a trade', 'look for job' and 'I am not sure'. These were coded as 'continue school' and 'not continue school.

\section{Health behaviour measures}

Physical activity

Physical activity was investigated by: 'How physically active are you?', response options were 'not physically active', 'a little physically active', 'physically active' and 'very physically active'. Responses were dichotomised into two categories of 'less physically active' ('not physically active', 'a little physically active') and 'physically active' ('physically active' and 'very physically active').

\section{Fruit intake}

Fruit intake was measured by: 'During the past one week ( $7 \mathrm{~d}$ ), on how many days did you usually eat fruit, such as oranges, pineapple, watermelon, banana, guava, pear, sweet apple, mangoes, or pawpaw?', response options were 'not once', '1-3d', '4-6d' and 'every day'. These were dichotomised into categories 'rarely' ( $\leq 3 \mathrm{~d} /$ week) and 'often' ( $\geq 4 \mathrm{~d} /$ week).

\section{Vegetable intake}

Vegetable intake was measured by: 'During the past $7 \mathrm{~d}$ on how many days did you usually eat vegetable such as kontomire, garden eggs, lettuce, cabbage, okra, alefu, bira, ayoyo, or bean leaves?', response options were 'not once', '1-3 d', '4-6 d' and 'every day'. The responses were categorised into dichotomous responses, 'rarely' ( $\leq 3 \mathrm{~d} /$ week) and 'often' ( $\geq 4 \mathrm{~d} /$ week).

\section{Breakfast eating}

Breakfast eating was measured by: 'During the past $7 \mathrm{~d}$ on how many days did you usually eat breakfast?', response options were 'not once', '1-3 d', '4-6 d' and 'every day'. The responses were categorised into dichotomous responses, 'rarely' ( $\leq 3 \mathrm{~d} /$ week) and 'often' ( $\geq 4 \mathrm{~d} /$ week).

Breakfast eating was further probed, to ascertain why adolescents do not skip breakfast, by the question: 'What is the main reason why you do not eat breakfast?' with the responses 'I always eat breakfast', 'I do not have time for breakfast', 'I cannot eat early in the morning', 'sometimes there is no food in my home' and 'some other reason, why?'

\section{Data analysis}

Pearson $\chi^{2}$ tests were used to test the associations between gender and each of the studied variables. Logistic regression analysis was used to model the associations between the socio-economic measures and the health behaviour variables. The strength of the associations was expressed by odds ratios and $95 \%$ confidence intervals.

Bivariate models were fitted including each of the socioeconomic measures one at a time, controlling for age and gender. Differences in breakfast eating, physical activity and fruit and vegetable intake by age group (12-15-year-olds v. 16-18-year-olds), gender, rural-urban residential area, school type (private $v$. public school) and regions (Eastern, Greater Accra and Volta) were also studied. Multivariate analyses were conducted for the SES indicators that were statistically significant at the bivariate level to ascertain their independent effect on the outcome measures. Interactions between SES and age and gender were investigated but no statistically significant interactions were found. In all analyses, those with the lowest SES were used as the reference category. Two-tailed $P$ value $<0.05$ was considered as statistically significant. The SPSS for Windows statistical software package version 16 (SPSS Inc., Chicago, IL, USA) was used for the analyses.

\section{Results}

Included in the present study were 498 boys ( $41.7 \%)$ and $659(55 \cdot 1 \%)$ girls; thirty-eight $(3 \cdot 2 \%)$ did not indicate their gender. The mean ages for boys and girls were $15 \cdot 7$ (SD 1.6) years and 15.8 (SD 1.6) years, respectively. Of all adolescents, 69\% were physically active, 56\% rarely ate fruits, $48 \%$ rarely ate vegetables and $32 \%$ rarely ate 


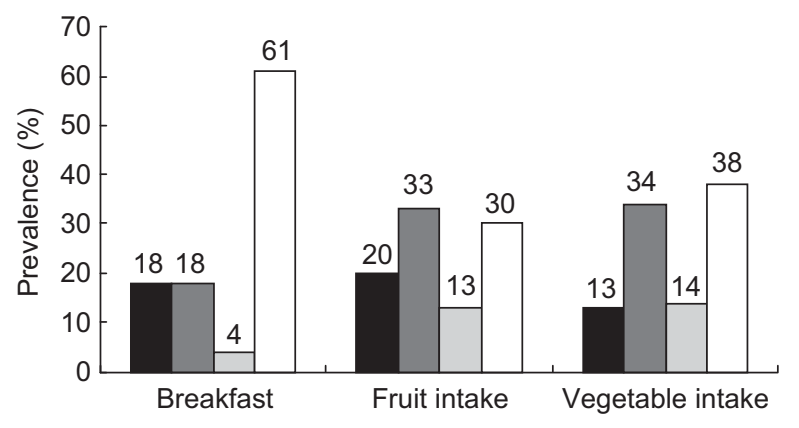

Fig. 1 Prevalence of breakfast eating and fruit and vegetable consumption in the past $7 \mathrm{~d}$ ( $\square$, not once; $\square, 1-3 \mathrm{~d}$; $\square, 4-6 \mathrm{~d}$; $\square$, every day) among 12-18-year-old Ghanaian adolescents, 2008

breakfast (Fig. 1). The differences by gender were statistically significant for the following variables: material affluence, family structure, father's and mother's education, mother's occupation, school performance, plans after graduation and physical activity (Table 1). We probed further to find out reasons why adolescents do not eat breakfast and the most commonly stated reason (50\%) was lack of food at home. Other reasons that were given included: 'I don't have time for breakfast' (24\%), 'I cannot eat early in the morning' (19\%) and 'some other reasons' (7\%).

Higher material affluence was indicative of higher physical activity and frequent breakfast intake (Table 2). Living in a nuclear family predicted frequent breakfast intake. Adolescents whose fathers had higher education were physically more active, ate more fruit and vegetables and more frequently took breakfast compared with their peers whose fathers had low education. Similarly, adolescents whose mothers had higher education were more physically active and ate breakfast frequently. Parental occupation was not related to any of the measured health behaviours; the only exception was that adolescents of mothers who had low grade jobs were more physically active compared with those whose mothers were unemployed. Higher school performance predicted higher fruit and vegetable intake (Table 2). On the other hand, having plans to continue studying after graduation was indicative of frequent breakfast intake.

When examined by age, it was found that younger adolescents (12-15-year-olds) were more likely to consume fruits and vegetables frequently than older ones (16-18-year-olds). Boys were more likely to participate in physical activity than girls (Table 2). There were no statistically significant gender differences in fruit and vegetable consumption. Adolescents in the private schools were more likely to participate in physical activity than those in public schools. No statistically significant rural-urban differences in any of the studied health behaviours were found, except that breakfast taking was more frequent among the rural adolescents compared with the urbaners. By region, those in Eastern and Greater
Accra regions were more likely to consume fruits and vegetables frequently than those in the Volta region.

In multivariate analyses (Table 3), the following were found. The probability of frequent breakfast eating was higher in adolescents from more affluent backgrounds than in those from less affluent ones. Compared with those whose mothers were illiterate, both tertiary and primary maternal educational attainment increased the probability of frequent fruit intake. Similarly, tertiary educational attainment increased the likelihood of frequent vegetable intake. Compared with those with unemployed mothers, mothers' low employment grade was related to higher probability of frequent fruit intake. High school performance was associated with frequent fruit intake, whereas high or medium school performance increased the likelihood of vegetable intake compared with low school performance.

\section{Discussion}

First, there were relatively low levels of breakfast eating, fruit and vegetable intake as well as physical activity among Ghanaian adolescents. Second, socio-economic differences were found. Higher father's educational level and greater material affluence were statistically significantly associated with more physical activity. Adolescent's own social position measured by school performance was the most statistically significant predictor of fruit and vegetable intake; compared with those with low school performance, those with higher school performance had frequent fruit and vegetable intake. Parental education, on the other hand, was the most statistically significant predictor of breakfast eating, so that those whose parents had higher educational levels ate breakfast frequently. Boys were more likely to participate in physical activity than girls. Family structure, father's occupation and adolescent's plans after graduation did not independently predict any of the outcome measures.

In Western countries low levels of breakfast intake among adolescents have been reported ${ }^{(5,6)}$. Similarly in Ghana, breakfast intake was such that $31 \%$ took breakfast less than four days in a week. In Western countries reasons for skipping breakfast included stress, lack of time, lack of parental control or a way of controlling body weight $^{(5,6)}$. In the present study, half of adolescents who did not take breakfast reported they did so because they had no food at home. In Ghana, as in most developing countries, there is huge poverty; it is estimated that $52 \%$ of the population is below the poverty line ${ }^{(18)}$. Many people are therefore likely rather to be concerned about what is available to eat, and planning what to eat and when to eat could be perhaps a 'luxury'. Thus whereas in Western countries adolescents skip breakfast to lose weight, their peers in some developing countries like Ghana skip breakfast because of lack of food at home. 
Table 1 Characteristics of the study participants by gender: 12-18-year-old Ghanaian adolescents, 2008

\begin{tabular}{|c|c|c|c|}
\hline & Boys ( $n$ 498) & Girls ( $n$ 659) & \\
\hline Variable & $\%$ & $\%$ & $P$ value (for gender difference) ${ }^{*}$ \\
\hline Age (years) & & & $0 \cdot 370$ \\
\hline 12 & $3 \cdot 0$ & $2 \cdot 3$ & \\
\hline 13 & $9 \cdot 2$ & $6 \cdot 5$ & \\
\hline 14 & $13 \cdot 7$ & $13 \cdot 1$ & \\
\hline 15 & $16 \cdot 7$ & $20 \cdot 2$ & \\
\hline 16 & $19 \cdot 1$ & $21 \cdot 1$ & \\
\hline 17 & $20 \cdot 5$ & $20 \cdot 9$ & \\
\hline 18 & $17 \cdot 9$ & $15 \cdot 9$ & \\
\hline Material affluence scale ( $n$ 1126) & & & $<0.001$ \\
\hline Poorest & $21 \cdot 9$ & $16 \cdot 5$ & \\
\hline Poor & $23 \cdot 1$ & $14 \cdot 7$ & \\
\hline Average & $20 \cdot 5$ & $19 \cdot 3$ & \\
\hline Affluent & $13 \cdot 9$ & $21 \cdot 9$ & \\
\hline Most affluent & $15 \cdot 7$ & $21 \cdot 2$ & \\
\hline Missing & $5 \cdot 0$ & $6 \cdot 4$ & \\
\hline Family structure ( $n$ 1189) & & & $0 \cdot 010$ \\
\hline Both parents dead & $5 \cdot 4$ & $2 \cdot 0$ & \\
\hline Only one parent alive & $17 \cdot 9$ & $16 \cdot 7$ & \\
\hline Both parents alive but not living together & $21 \cdot 9$ & $21 \cdot 2$ & \\
\hline Nuclear family & $54 \cdot 4$ & $59 \cdot \overline{5}$ & \\
\hline Missing & $0 \cdot 4$ & 0.6 & \\
\hline Father's education ( $n$ 1133) & & & 0.008 \\
\hline Illiterate & $10 \cdot 4$ & $6 \cdot 5$ & \\
\hline Primary education & $34 \cdot 3$ & $29 \cdot 0$ & \\
\hline Secondary education & $27 \cdot 9$ & $33 \cdot 2$ & \\
\hline Tertiary education & $22 \cdot 3$ & $25 \cdot 8$ & \\
\hline Missing & $5 \cdot 0$ & $5 \cdot 5$ & \\
\hline Mother's education ( $n$ 1146) & & & 0.001 \\
\hline Illiterate & $18 \cdot 5$ & $14 \cdot 7$ & \\
\hline Primary education & $46 \cdot 6$ & $39 \cdot 8$ & \\
\hline Secondary education & $22 \cdot 9$ & $30 \cdot 7$ & \\
\hline Tertiary education & $7 \cdot 2$ & $11 \cdot 2$ & \\
\hline Missing & $4 \cdot 8$ & $3 \cdot 6$ & \\
\hline Father's occupation ( $n$ 1048) & & & 0.060 \\
\hline Unemployed & 3.8 & 3.6 & \\
\hline Low grade & $61 \cdot 8$ & $52 \cdot 4$ & \\
\hline High grade & $25 \cdot 3$ & $29 \cdot 7$ & \\
\hline Missing & $9 \cdot 0$ & $14 \cdot 3$ & \\
\hline Mother's occupation ( $n$ 1120) & & & 0.017 \\
\hline Unemployed & $4 \cdot 4$ & 3.0 & \\
\hline Low grade & $82 \cdot 1$ & $77 \cdot 2$ & \\
\hline High grade & $8 \cdot 0$ & $12 \cdot 7$ & \\
\hline Missing & $5 \cdot 4$ & $7 \cdot 0$ & \\
\hline School performance ( $n$ 1188) & & & $<0.001$ \\
\hline Low & $8 \cdot 6$ & $16 \cdot 5$ & \\
\hline Middle & $48 \cdot 4$ & $54 \cdot 2$ & \\
\hline High & $42 \cdot 2$ & $28 \cdot 8$ & \\
\hline Missing & $0 \cdot 8$ & 0.5 & \\
\hline Plans after graduation ( $n$ 1186) & & & 0.028 \\
\hline Not continue school & $14 \cdot 3$ & $10 \cdot 2$ & \\
\hline Continue school & $84 \cdot 3$ & $89 \cdot 5$ & \\
\hline Missing & $1 \cdot 4$ & 0.3 & \\
\hline
\end{tabular}

*Missing values were excluded from the statistical test.

Material affluence and parental educational levels were associated with breakfast eating so that those from advantaged backgrounds ate breakfast frequently, although the association was weak. The relationship between material affluence and breakfast eating was not surprising given that half of those who skipped breakfast cited lack of food at home as the reason for doing so. Other studies have also found that frequent breakfast taking was typical of adolescents of higher SES ${ }^{(5,6,19,20)}$. More studies are needed in developing countries to investigate whether skipping breakfast is associated with health-compromising behaviours among adolescents as has been reported in some Western countries ${ }^{(7,20)}$ or whether it is just a meal timing preference or related to poverty.

Despite the well-known advantages of fruit and vegetable consumption ${ }^{(8,9)}$, low fruit and vegetable consumption was found among Ghanaian adolescents. Overall, only about a third of adolescents ate fruit or vegetables 
Table 2 Odds ratios and $95 \%$ confidence intervals for breakfast eating, fruit and vegetable consumption and physical activity by socio-economic measures, adjusted for age and gender: 12-18-year-old Ghanaian adolescents, 2008

\begin{tabular}{|c|c|c|c|c|c|c|c|c|}
\hline \multirow[b]{2}{*}{ Socio-economic indicator } & \multicolumn{2}{|c|}{ Breakfast eating } & \multicolumn{2}{|c|}{ Fruit intake } & \multicolumn{2}{|c|}{ Vegetable intake } & \multicolumn{2}{|c|}{ Physical activity } \\
\hline & OR & $95 \% \mathrm{Cl}$ & OR & $95 \% \mathrm{Cl}$ & OR & $95 \% \mathrm{Cl}$ & OR & $95 \% \mathrm{Cl}$ \\
\hline \multicolumn{9}{|l|}{ Age } \\
\hline 12-15-year-olds & $1 \cdot 0$ & & $1 \cdot 0$ & & $1 \cdot 0$ & & $1 \cdot 0$ & \\
\hline $16-18$ year-olds & 0.9 & $0 \cdot 7,1 \cdot 1$ & 0.8 & $0.6,0.9$ & 0.6 & $0.4,0.7$ & $1 \cdot 1$ & $0.8,1 \cdot 4$ \\
\hline \multicolumn{9}{|l|}{ Gender } \\
\hline Boys & $1 \cdot 0$ & & $1 \cdot 0$ & & $1 \cdot 0$ & & $1 \cdot 0$ & \\
\hline Girls & $1 \cdot 2$ & $1 \cdot 0,1 \cdot 6$ & 0.8 & $0 \cdot 6,1 \cdot 0$ & 0.9 & $0 \cdot 7,1 \cdot 1$ & 0.6 & $0.5,0.8$ \\
\hline \multicolumn{9}{|l|}{ Place of residence } \\
\hline Rural & $1 \cdot 0$ & & $1 \cdot 0$ & & $1 \cdot 0$ & & $1 \cdot 0$ & \\
\hline Urban & 0.7 & $0.5,0.9$ & 0.8 & $0 \cdot 6,1 \cdot 0$ & $1 \cdot 0$ & $0 \cdot 8,1 \cdot 3$ & $1 \cdot 0$ & $0 \cdot 8,1 \cdot 3$ \\
\hline \multicolumn{9}{|l|}{ School type } \\
\hline Private & $1 \cdot 0$ & & $1 \cdot 0$ & & $1 \cdot 0$ & & $1 \cdot 0$ & \\
\hline Public & 0.8 & $0 \cdot 6,1 \cdot 1$ & 0.9 & $0 \cdot 7,1 \cdot 1$ & 0.8 & $0 \cdot 6,1 \cdot 0$ & 0.7 & $0.5,0.9$ \\
\hline \multicolumn{9}{|l|}{ Region } \\
\hline Volta & $1 \cdot 0$ & & $1 \cdot 0$ & & $1 \cdot 0$ & & $1 \cdot 0$ & \\
\hline Eastern & $1 \cdot 2$ & $0.8,1 \cdot 6$ & $1 \cdot 1$ & $0 \cdot 8,1 \cdot 4$ & $1 \cdot 7$ & $1 \cdot 3,2 \cdot 3$ & 0.9 & $0 \cdot 7,1 \cdot 2$ \\
\hline Greater Accra & 0.8 & $0 \cdot 6,1 \cdot 0$ & 0.8 & $0 \cdot 6,1 \cdot 1$ & $1 \cdot 4$ & $1 \cdot 1,1 \cdot 8$ & $1 \cdot 0$ & $0 \cdot 7,1 \cdot 3$ \\
\hline \multicolumn{9}{|l|}{ Family structure } \\
\hline Both parents dead & $1 \cdot 0$ & & $1 \cdot 0$ & & $1 \cdot 0$ & & $1 \cdot 0$ & \\
\hline One parent alive & $1 \cdot 7$ & $0.8,3 \cdot 4$ & $1 \cdot 0$ & $0 \cdot 5,2 \cdot 0$ & $1 \cdot 0$ & $0.5,2 \cdot 0$ & 1.6 & $0.8,3 \cdot 2$ \\
\hline Both parents alive but not living together & $1 \cdot 8$ & $0.9,3.5$ & $1 \cdot 0$ & $0.5,1.9$ & $1 \cdot 2$ & $0 \cdot 6,2 \cdot 4$ & $1 \cdot 8$ & $0.9,3.6$ \\
\hline Nuclear family & $2 \cdot 2$ & $1 \cdot 2,4 \cdot 3$ & $1 \cdot 1$ & $0 \cdot 6,2 \cdot 0$ & $1 \cdot 4$ & $0 \cdot 7,2 \cdot 6$ & 1.9 & $1 \cdot 0,3 \cdot 7$ \\
\hline \multicolumn{9}{|l|}{ Material affluence scale } \\
\hline Poorest & $1 \cdot 0$ & & $1 \cdot 0$ & & $1 \cdot 0$ & & $1 \cdot 0$ & \\
\hline Poor & $1 \cdot 2$ & $0 \cdot 8,1 \cdot 8$ & $1 \cdot 4$ & $0.9,2 \cdot 0$ & $1 \cdot 1$ & $0.7,1 \cdot 6$ & 0.9 & $0.6,1 \cdot 4$ \\
\hline Average & $1 \cdot 8$ & $1 \cdot 3,2 \cdot 7$ & $1 \cdot 4$ & $1 \cdot 0,2 \cdot 1$ & $1 \cdot 3$ & $0.9,1.9$ & $1 \cdot 2$ & $0.8,1.9$ \\
\hline Affluent & $2 \cdot 8$ & $1 \cdot 9,4 \cdot 3$ & $1 \cdot 4$ & $1 \cdot 0,2 \cdot 1$ & $1 \cdot 4$ & $0 \cdot 9,2 \cdot 1$ & $2 \cdot 0$ & $1 \cdot 3,3 \cdot 1$ \\
\hline Most affluent & $3 \cdot 0$ & $2 \cdot 0,4 \cdot 5$ & $1 \cdot 0$ & $0 \cdot 7,1 \cdot 5$ & 0.8 & $0.6,1 \cdot 2$ & $2 \cdot 0$ & $1 \cdot 3,3 \cdot 0$ \\
\hline \multicolumn{9}{|l|}{ Father's education } \\
\hline Illiterate & $1 \cdot 0$ & & $1 \cdot 0$ & & $1 \cdot 0$ & & $1 \cdot 0$ & \\
\hline Primary education & $1 \cdot 2$ & $0 \cdot 8,2 \cdot 0$ & $1 \cdot 8$ & $1 \cdot 2,3 \cdot 1$ & $1 \cdot 2$ & $0 \cdot 8,2 \cdot 0$ & $1 \cdot 2$ & $0 \cdot 7,1 \cdot 8$ \\
\hline Secondary education & $1 \cdot 7$ & $1 \cdot 1,2 \cdot 7$ & 1.5 & $0.9,2.4$ & $1 \cdot 0$ & $0 \cdot 6,1 \cdot 5$ & $2 \cdot 6$ & $1 \cdot 6,4 \cdot 3$ \\
\hline Tertiary education & $3 \cdot 4$ & $2 \cdot 1,5 \cdot 8$ & 1.9 & $1 \cdot 2,3 \cdot 2$ & $1 \cdot 8$ & $1 \cdot 1,2 \cdot 9$ & $1 \cdot 8$ & $1 \cdot 1,3 \cdot 0$ \\
\hline \multicolumn{9}{|l|}{ Mother's education } \\
\hline Illiterate & $1 \cdot 0$ & & $1 \cdot 0$ & & $1 \cdot 0$ & & $1 \cdot 0$ & \\
\hline Primary education & $1 \cdot 3$ & $0.9,1 \cdot 8$ & $1 \cdot 3$ & $1 \cdot 0,1 \cdot 9$ & $1 \cdot 1$ & $0.8,1.5$ & 0.9 & $0 \cdot 6,1 \cdot 4$ \\
\hline Secondary education & $2 \cdot 5$ & $1 \cdot 7,3 \cdot 7$ & $1 \cdot 2$ & $0.9,1.8$ & $1 \cdot 3$ & $0 \cdot 9,1 \cdot 9$ & $1 \cdot 3$ & $0.8,1 \cdot 9$ \\
\hline Tertiary education & $3 \cdot 6$ & $2 \cdot 0,6 \cdot 5$ & $2 \cdot 0$ & $1 \cdot 2,3 \cdot 3$ & $1 \cdot 1$ & $0.7,1.9$ & $1 \cdot 0$ & $0.6,1 \cdot 8$ \\
\hline \multicolumn{9}{|l|}{ Father's occupation } \\
\hline Unemployed & $1 \cdot 0$ & & $1 \cdot 0$ & & $1 \cdot 0$ & & $1 \cdot 0$ & \\
\hline Low grade & $1 \cdot 1$ & $0 \cdot 6,2 \cdot 0$ & $1 \cdot 8$ & $0.9,3 \cdot 6$ & 0.9 & $0.5,1 \cdot 8$ & $1 \cdot 0$ & $0.5,2 \cdot 0$ \\
\hline High grade & $1 \cdot 8$ & $0.9,3.5$ & $1 \cdot 8$ & $0.9,3.7$ & 0.9 & $0.8,0.9$ & $1 \cdot 8$ & $0.9,3.6$ \\
\hline \multicolumn{9}{|l|}{ Mother's occupation } \\
\hline Unemployed & $1 \cdot 0$ & & $1 \cdot 0$ & & $1 \cdot 0$ & & $1 \cdot 0$ & \\
\hline Low grade & 0.8 & $0 \cdot 4,1 \cdot 6$ & $2 \cdot 3$ & $1 \cdot 1,4 \cdot 6$ & $1 \cdot 8$ & $0 \cdot 9,3 \cdot 4$ & 0.8 & $0 \cdot 4,1 \cdot 6$ \\
\hline High grade & $1 \cdot 2$ & $0 \cdot 6,2 \cdot 7$ & 1.9 & $0.9,4 \cdot 2$ & $1 \cdot 3$ & $0 \cdot 6,2 \cdot 7$ & $1 \cdot 2$ & $0.5,2 \cdot 7$ \\
\hline \multicolumn{9}{|l|}{ School performance } \\
\hline Low & $1 \cdot 0$ & & $1 \cdot 0$ & & $1 \cdot 0$ & & $1 \cdot 0$ & \\
\hline Medium & $1 \cdot 2$ & $0 \cdot 8,1 \cdot 7$ & $1 \cdot 6$ & $1 \cdot 1,2 \cdot 4$ & $1 \cdot 6$ & $1 \cdot 2,2 \cdot 4$ & $1 \cdot 0$ & $0.7,1.5$ \\
\hline High & $1 \cdot 3$ & $0.9,2 \cdot 0$ & $2 \cdot 9$ & $1 \cdot 9,4 \cdot 4$ & $2 \cdot 3$ & $1 \cdot 5,3 \cdot 4$ & $1 \cdot 4$ & $0 \cdot 9,2 \cdot 1$ \\
\hline \multicolumn{9}{|l|}{ Plans after graduation } \\
\hline Not continue school & $1 \cdot 0$ & & $1 \cdot 0$ & & $1 \cdot 0$ & & $1 \cdot 0$ & \\
\hline Continue school & $1 \cdot 6$ & $1 \cdot 1,2 \cdot 3$ & 0.8 & $0 \cdot 5,1 \cdot 1$ & $1 \cdot 2$ & $0 \cdot 8,1 \cdot 7$ & $1 \cdot 2$ & $0 \cdot 8,1 \cdot 8$ \\
\hline
\end{tabular}

daily while less than $50 \%$ of all adolescents ate vegetables or fruits more than four days a week. In their review of the determinants of adolescents' fruit and vegetable consumption, Rasmussen et al. ${ }^{(21)}$ found that socio-economic position is a consistent determinant of adolescent fruit and vegetable intake. The association between father's education and frequent fruit intake disappeared when the other SES indicators were controlled for in the present study. However, higher mother's education predicted frequent fruit intake while those whose mothers had a low grade occupation frequently consumed fruit compared with adolescents of unemployed mothers. Similar associations of parental SES and fruit and vegetable consumption have been found in other non-Western countries ${ }^{(22,23)}$. In Ghana, fruits and vegetables are readily available and cheap, especially during the main harvest seasons from June to August. This could therefore explain why material affluence has no effect on the frequency of consumption. 
Table 3 Odds ratios and $95 \%$ confidence intervals for breakfast eating, fruit and vegetable consumption and physical activity by socioeconomic measures, in a multivariate model: 12-18-year-old Ghanaian adolescents, 2008

\begin{tabular}{|c|c|c|c|c|c|c|c|c|}
\hline \multirow[b]{2}{*}{ Socio-economic indicator } & \multicolumn{2}{|c|}{ Breakfast eating } & \multicolumn{2}{|c|}{ Fruit intake } & \multicolumn{2}{|c|}{ Vegetable intake } & \multicolumn{2}{|c|}{ Physical activity } \\
\hline & OR & $95 \% \mathrm{Cl}$ & OR & $95 \% \mathrm{Cl}$ & OR & $95 \% \mathrm{Cl}$ & OR & $95 \% \mathrm{Cl}$ \\
\hline Family structure ( $n$ 1189) & & & & * & & * & & * \\
\hline Both parents dead & $1 \cdot 0$ & & & & & & & \\
\hline One parent alive & 0.8 & $0 \cdot 4,1 \cdot 7$ & & & & & & \\
\hline Both parents alive but not living together & $0 \cdot 8$ & $0 \cdot 6,1 \cdot 2$ & & & & & & \\
\hline Nuclear family & $1 \cdot 0$ & $0 \cdot 7,1 \cdot 4$ & & & & & & \\
\hline Material affluence scale ( $n$ 1126) & & & & * & & * & & \\
\hline Poorest & $1 \cdot 0$ & & & & & & $1 \cdot 0$ & \\
\hline Poor & $1 \cdot 0$ & $0 \cdot 6,1 \cdot 4$ & & & & & $1 \cdot 0$ & $0 \cdot 7,1 \cdot 5$ \\
\hline Average & $1 \cdot 5$ & $0 \cdot 9,2 \cdot 3$ & & & & & $1 \cdot 2$ & $0 \cdot 8,1 \cdot 8$ \\
\hline Affluent & $1 \cdot 8$ & $1 \cdot 1,2 \cdot 8$ & & & & & $1 \cdot 1$ & $0 \cdot 7,1 \cdot 7$ \\
\hline Most affluent & $1 \cdot 7$ & $1 \cdot 1,2 \cdot 7$ & & & & & 0.7 & $0 \cdot 5,1 \cdot 1$ \\
\hline \multicolumn{9}{|l|}{ Father's education ( $n$ 1133) } \\
\hline Illiterate & $1 \cdot 0$ & & $1 \cdot 0$ & & $1 \cdot 0$ & & $1 \cdot 0$ & \\
\hline Primary education & 0.9 & $0 \cdot 6,1 \cdot 6$ & $1 \cdot 7$ & $1 \cdot 1,2 \cdot 8$ & $1 \cdot 3$ & $0 \cdot 8,2 \cdot 0$ & $1 \cdot 2$ & $0 \cdot 7,2 \cdot 0$ \\
\hline Secondary education & $1 \cdot 0$ & $0.6,1 \cdot 8$ & $1 \cdot 3$ & $0 \cdot 7,2 \cdot 1$ & $1 \cdot 0$ & $0.6,1.6$ & $1 \cdot 0$ & $0.8,1 \cdot 7$ \\
\hline Tertiary education & 1.9 & $1 \cdot 1,3 \cdot 5$ & $1 \cdot 5$ & $0.9,2.6$ & $1 \cdot 9$ & $1 \cdot 2,3 \cdot 1$ & $1 \cdot 8$ & $1 \cdot 0,3 \cdot 1$ \\
\hline \multicolumn{9}{|l|}{ Mother's education ( $n$ 1146) } \\
\hline Illiterate & $1 \cdot 0$ & & $1 \cdot 0$ & & $1 \cdot 0$ & & $1 \cdot 0$ & \\
\hline Primary education & $1 \cdot 0$ & $0 \cdot 7,1 \cdot 5$ & $1 \cdot 5$ & $1 \cdot 1,2 \cdot 1$ & & & $1 \cdot 1$ & $0 \cdot 7,1 \cdot 5$ \\
\hline Secondary education & $1 \cdot 4$ & $0.9,2.3$ & $1 \cdot 3$ & $0 \cdot 9,2 \cdot 1$ & & & $1 \cdot 2$ & $0.8,1.9$ \\
\hline Tertiary education & $1 \cdot 8$ & $1 \cdot 0,3 \cdot 4$ & $2 \cdot 3$ & $1 \cdot 2,4 \cdot 1$ & & & $1 \cdot 1$ & $0.6,1.9$ \\
\hline \multicolumn{9}{|l|}{ Mother's occupation ( $n$ 1120) } \\
\hline Unemployed & & & $1 \cdot 0$ & & & & & \\
\hline Low grade & & & $2 \cdot 3$ & $1 \cdot 1,4 \cdot 9$ & & & & \\
\hline High grade & & & $1 \cdot 7$ & $0 \cdot 7,3 \cdot 9$ & & & & \\
\hline School performance ( $n$ 1188) & & & & & & & & * \\
\hline Low & $1 \cdot 0$ & & $1 \cdot 0$ & & $1 \cdot 0$ & & & \\
\hline Medium & $1 \cdot 2$ & $0 \cdot 8,1 \cdot 7$ & $1 \cdot 4$ & $0 \cdot 9,2 \cdot 1$ & $1 \cdot 6$ & $1 \cdot 1,2 \cdot 4$ & & \\
\hline High & $1 \cdot 3$ & $0.9,2 \cdot 0$ & $2 \cdot 4$ & $1 \cdot 5,3 \cdot 7$ & $2 \cdot 1$ & $1 \cdot 4,3 \cdot 2$ & & \\
\hline \multicolumn{9}{|l|}{ Plans after graduation ( $n$ 1186) } \\
\hline Not continue school & $1 \cdot 0$ & & & & & & & \\
\hline Continue school & 1.5 & $1 \cdot 0,2 \cdot 2$ & & & & & & \\
\hline
\end{tabular}

${ }^{*}$ Not included in the analyses.

One interesting finding was that an adolescent's own social position measured by school performance seems to be the most important predictor of fruit and vegetable consumption. This could mean that adolescents with better school performance are also the most informed about health issues like the health benefits of fruit and vegetable consumption and thus are most likely to adopt health-enhancing behaviours. However, we cannot rule out the role of the interplay of socio-cultural factors in fruit and vegetable consumption.

Low physical activity has been reported in previous research $^{(24-26)}$, as in ours. Several other studies found positive associations between SES and physical activity among adolescents ${ }^{(12,27)}$. In South Africa for example, McVeigh et $a l .{ }^{(13)}$ reported that mother's education was positively associated with physical activity among adolescents. Low physical activity among adolescents in developing countries like Ghana is likely to be more related to the availability of facilities at the societal level. In Ghana for example, the design of residential areas is such that walk ways and bicycle lanes are not available. Social and welfare centres do not have such facilities either. Besides, due to rapid urbanisation most spaces that serve as playgrounds and football fields are all being filled with houses and shops. These factors are likely to inhibit participation in physical activities. No independent association was found between physical activity and any of the SES measures in our study. Similarly, some of the previous studies reviewed by Hanson and Chen ${ }^{(12)}$ also found a null association between physical activity and parental education, household income and school district SES.

Gender differences in physical activity were found among adolescents such that boys had slightly higher participation in physical activity compared with girls. This is consistent with previous studies ${ }^{(12,27)}$. One plausible explanation could be related to culture. Sporting activities are generally perceived as masculine activities so this could be a disincentive for girls. This cultural aspect needs to be investigated further.

The present study design being cross-sectional means that the cause-and-effect relationship cannot be emphasised as a causal conclusion. However, it is likely that all of the socio-economic measures investigated preceded in time, rather than were followed by, the adolescents' health behaviours. The covariates in analyses are likely to affect the power of the study, despite its useful findings. Data were self-reported and no validation studies have 
been conducted to assess the validity of the responses. However, relationships between SES and health/health behaviours among adolescents have been extensively studied $^{(12,28)}$. Due to scarce resources, only adolescents in schools were included in the present study, and hence the results reported herein might not necessarily be same in the entire adolescent population. Although the school enrolment rate in Ghana is relatively high $(78.8 \%$ for Junior High Schools ${ }^{(14)}$ ), given that the survey was mainly conducted in schools still limits its generalisability to non-students.

A validation study which would examine inter-rater and test-retest reliability of adolescent SES as well as health behaviours in developing countries would give more insight into researching adolescent health inequalities in such countries. Our report of fruit and vegetable intake could be affected by seasonal variation in availability and cost. However, as the survey was done during the peak harvest season (June-August) it is likely that the prevalence of fruit and vegetable intake reported was much higher than during other periods of the year. Nevertheless, our study provides insight into the extent to which SES differences are related to health behaviours in a developing country setting.

Action should be taken to improve breakfast eating, fruit and vegetable intake and physical activity in order to prevent degenerative diseases among Ghanaian adolescents. Health promotion that aims at increasing breakfast intake should have as a target increasing knowledge as well as food accessibility to adolescents. Increasing fruit and vegetable consumption as well as breakfast intake can be done by serving fruit and vegetables during school meals and by introduction of free meals in all Junior and Senior High Schools, as has been started in a few rural schools. Activities that would enhance knowledge on the health benefits of consumption of fruits and vegetables should target the whole adolescent population but pay more attention to those with low parental educational background and those whose school performance is low. Interventions are needed to promote physical activity, especially those that would increase accessibility to playgrounds and encouragement of girls' participation. Since physical activity is a cross-cutting issue, the design of cities and roads should aim at providing, for example, safe walk ways and bicycle lanes.

\section{Conclusions}

The main conclusion from the present study is that breakfast eating, fruit and vegetable intake and physical activity were relatively low among Ghanaian adolescents. Moreover, there were socio-economic differences in all of the studied health behaviours.

Overall, it seems that there may be factors other than those considered here which also relate to the studied behaviours. Interventions are needed to improve breakfast eating, fruit and vegetable intake and physical activity in order to prevent degenerative diseases adolescents. Socio-economic differences should be taken into account when developing interventions.

\section{Acknowledgements}

The study was supported by the Finnish Cultural Foundation Central Fund; the Ministry of Social Affairs and Health, Finland; the Juho Vainio Foundation; and the Competitive Research Funding of the Pirkanmaa Hospital District, Tampere University Hospital (Grant 9J104). The authors declare that they have no conflict of interest. D.D. conceived of and designed the study. D.D., S.R. and A.R. were involved in the drafting and the revising of the questionnaire. D.D. was the principal investigator during the data collection. D.D. analysed the data and drafted the manuscript. All authors were involved in the interpretation of data and the critical revision of the manuscript for important intellectual content. All authors gave final approval of the version to be published. The authors extend many thanks to the WHO Country Office, Ghana, particularly Mr Selassi D'Amanda, for the provision of office space and technical support during the data collection. Their appreciation also goes to the Ghana Health Service, Adolescents Health Unit, for providing the vehicle for the data collection. They would like to thank Mr Charles Fenuku, Mr Akuffo, Miss Forzia, Miss Tani Abubakari, Miss Rejoice Nutakor, Mrs Cynthia Bosumtwi-Sam, Miss Rose, $\mathrm{Mr}$ Boahen, and Mr Francis Kpodo and his team for assisting in data collection and entry; and Mr Lasse Pere for data management.

\section{References}

1. Prentice MA (2006) The emerging epidemic of obesity in developing countries. Int J Epidemiol 35, 93-99.

2. Pampel FC (2005) Patterns of tobacco use in the early epidemic stages: Malawi and Zambia. Am J Public Health 95, 1009-1015.

3. Warburton DE, Nicol CW \& Bredin SS (2006) Health benefits of physical activity: the evidence. CMAJ 174, 801-809.

4. Harrison PA \& Narayan G (2003) Differences in behavior, psychological factors, and environmental factors associated with participation in school sports and other activities in adolescence. $J$ Sch Health 73, 113-120.

5. Nicklas TA, O'Niel C \& Meyers L (2004) The importance of breakfast consumption to nutrition of children, adolescents, and young adults. Nutr Today 39, 30-39.

6. Mullie P, Clarys P, De Ridder D et al. (2006) Breakfast frequency and fruit and vegetable consumption in Belgian adolescents. Nutr Food Sci 36, 315-326.

7. Keski-Rahkonen A, Kaprio J, Rissanen A et al. (2003) Breakfast skipping and health-compromising behaviors in adolescents and adults. Eur J Clin Nutr 57, 842-853.

8. World Cancer Research Fund (1997) Food, Nutrition and the Prevention of Cancer: A Global Perspective. Washington, DC: American Institute for Cancer Research. 
9. Tylavsky FA, Holliday K, Danish R et al. (2004) Fruit and vegetable intakes are an independent predictor of bone size in early pubertal children. Am J Clin Nutr 79, 311-317.

10. Hirvensalo M \& Lintunen T (2011) Life-course perspective for physical activity and sports participation. Eur Rev Aging Phys Act 8, 13-22.

11. Chiolero A \& Schmid H (2002) Repeated self-reported injuries and substance use among young adolescents; the case of Switzerland. Soz Praventivmed 47, 289-297.

12. Hanson MD \& Chen E (2007) Socioeconomic status and health behaviors in adolescence: a review of the literature. J Behav Med 30, 263-285.

13. McVeigh JA, Norris SA \& deWet T (2004) The relationship between socioeconomic status and physical activity patterns in South African Children. Acta Paediatr 93, 982-988.

14. Ministry of Education, Science and Sport, Ghana (2008) Reports on basic statistics and planning parameters for basic education in Ghana 2007/2008. EMIS Project, p. 16. Accra: Ministry of Education, Science and Sport, Ghana Publications.

15. Doku D, Koivusilta L \& Rimpelä A (2010) Indicators for measuring material affluence of adolescents in health inequality research in developing countries. Child Ind Res 3, 243-260

16. Townsend P (1987) Deprivation. J Soc Policy 16, 125-146.

17. Currie C, Molcho M, Boyce W et al. (2008) Researching health inequalities in adolescents: the development of the Health Behaviour in School-Aged Children (HBSC). Soc Sci Med 6, 1429-1436.

18. Coulombe H \& McKay A (2004) Selective poverty reduction in a slow growth environment: Ghana in the 1990s. In Globalization, Employment and Poverty in Ghana [E Aryeetey, editor]. http://www.rrojasdatabank.info/widerconf/Aryeetey. pdf (accessed January 2011).
19. O'Dea JA \& Caputi P (2001) Association between socioeconomic status, weight, age and gender, and the body image and weight control practices of 6- to 19-year-old children and adolescents. Health Educ Res 16, 521-532.

20. Höglund D, Samuelson G \& Mark A (1998) Food habits in Swedish adolescents in relation to socioeconomic conditions. Eur J Clin Nutr 52, 784-789.

21. Rasmussen M, Krølner R, Klepp K et al. (2006) Determinants of fruit and vegetable consumption among children and adolescents: a review of the literature. Part I: quantitative studies. Int J Behav Nutr Phys Act 3, 22.

22. Wang Y, Bentley ME, Fengying Z et al. (2002) Tracking of dietary intake patterns of Chinese from childhood to adolescence over a six-year follow-up period. J Nutr $\mathbf{1 3 2}$, 430-438.

23. Sabbah AH, Vereecken C, Kolsteren P et al. (2007) Food habits and physical activity patterns among Palestinian adolescents: findings from the national study of Palestinian schoolchildren (HBSC-WBG2004). Public Health Nutr 10, 739-746.

24. World Health Organization (2004) Global Strategy on Diet, Physical Activity and Health. Physical Activity and Young People. Geneva: WHO; available at http://www.who.int/ dietphysicalactivity/factsheet_young_people/en/index.html

25. Heath G, Pratt M, Warren C et al. (1994) Physical activity patterns in American high school students. Arch Pediatr Adolesc Med 148, 1131-1136.

26. Trippe H (1996) Children and sport. Br Med J312, 199-200.

27. Ferreira I, van der Horst K, Wendel-Vos W et al. (2007) Environmental correlates of physical activity in youth $-\mathrm{a}$ review and update. Obes Rev $\mathbf{8}, 129-154$.

28. Koivusilta LK, Rimpela AH \& Kautiainen SM (2006) Health inequality in adolescence. Does stratification occur by familial social background, family affluence, or personal social position? BMC Public Health 6, 110. 